\title{
La résistance du lexical à la dématérialisation : l'exemple des adverbes intensifs awfully, frightfully, terribly
}

\section{Christopher Desurmont}

\section{(2) OpenEdition \\ Journals}

Édition électronique

URL : http://journals.openedition.org/esa/1637

DOI : $10.4000 /$ esa. 1637

ISSN : 2650-2623

Éditeur

Société de stylistique anglaise

Édition imprimée

Date de publication : 31 décembre 2011

Pagination : 123-130

ISSN : 2116-1747

\section{Référence électronique}

Christopher Desurmont, «La résistance du lexical à la dématérialisation : l'exemple des adverbes intensifs awfully, frightfully, terribly », Études de stylistique anglaise [En ligne], 3 | 2011, mis en ligne le 27 novembre 2018, consulté le 02 mai 2019. URL : http://journals.openedition.org/esa/1637 ; DOI : 10.4000/esa. 1637 


\title{
LA RÉSISTANCE DU LEXICAL À LA DÉMATÉRIALISATION : L'EXEMPLE DES ADVERBES INTENSIFS AWFULLY, FRIGHTFULLY, TERRIBLY ${ }^{1}$
}

\author{
Christopher Desurmont \\ Université Lille 3, STL, UMR 8163
}

\begin{abstract}
The common denominator of the three intensive adverbs here examined is the high degree of intensity of the emotion denoted by their nominal roots, enabling these adverbs to be used as scalar modifiers (boosters). This paper shows that the semantic component of these intensifiers is often barely perceptible, but can be made to reappear in appropriate contexts: the adjectival phrase (adverb plus adjective) can then allow a cause and effect interpretation through a right-to-left reading of the AP; or a metalinguistic interpretation when the meaning of the adverb is perceived as one of the semantic components of the adjective. Elsewhere, an oxymoron (adverb + adjective) is created in the first of two coordinate clauses, undermining the adjectival property and paving the way for a second clause with implicit negation of this adjectival predication : a reversal which, it is claimed, is disallowed if adverbial modification of the adjective is done with a maximizer.
\end{abstract}

Keywords: adjective, adverb, bleaching, interpretation, scalarity

1 Cet article, accepté par le comité de lecture, est issu de la communication présentée en mai 2011 dans l'atelier de stylistique lors du $51^{\text {ème }}$ Congrès de la SAES à Paris Diderot et Paris Sorbonne Nouvelle. 
1. Les adverbes awfully, frightfully, terribly (A, F, T) sont des « adverbes intensifs » appartenant à la classe des «modificateurs scalaires» (Scalar modifiers) de type booster, catégorie comprenant aussi par exemple extremely, highly, very, terrifically ${ }^{2}$. Portant sur des adjectifs, A, F et T sont donc des «modificateurs de degré » de la qualité adjectivale. Ces adverbes sont construits sur une base adjectivale dont la racine dénote un «sentiment » caractérisé par un degré élevé d'intensité. Cette dimension quantitative explique que ces adverbes puissent servir comme quantificateurs dans l'expression du «haut degré » de la qualité adjectivale.

2. Divers indices témoignent du degré élevé de dématérialisation de ces adverbes de « sentiments » :

a) l'existence de nombreuses expressions courantes telles que awfully kind of you, frightfully hot, terribly important ;

b) les commutations possibles entre ces adverbes ${ }^{3}$ :

(1) KBK 7007 Hopefully it's not terribly expensive.

(2) KCV 5287 Yeah it's awfully expensive

(3) KRX 79 I don't wish to interrupt you, I'm awfully sorry!

(4) CKF 860 'I'm terribly sorry to butt in like this.';

c) le fait que $\mathrm{A}, \mathrm{F}$ ou $\mathrm{T}$ sont compatibles avec une propriété positive :

(5) F9R 2089 'History is going to be my subject; modern history. Of course it's a frightfully easy degree, but it happens to be what I'm most interested in';

d) l'impossibilité de retrouver le signifié lexical de l'adverbe dans bien des contextes :

(6) GUS 552 And then she either becomes terribly unfashionable, or wears very expensive hats that another woman's husband has to pay for.'

En principe, une femme ne déclenche pas un sentiment de «terreur » par le simple fait d'être démodée.

e) l'emploi d'un même adverbe devant deux adjectifs de sens opposé :

2 Nous reprenons la division désormais classique des adverbes intensifs en deux catégories : d'une part les TOTALITY MODIFIERS comprenant les Maximizers (absolutely, completely, entirely, perfectly, totally, utterly ...) et les Approximators (almost, nearly, practically, virtually...) ; d'autre part les SCALAR MODIFIERS (Boosters, Moderators, Diminishers).

3 Exemples tirés du British National Corpus. 
(7) $\underline{\text { H8B }} 318$ 'How frightfully interesting', she said when Julia had finished';

(8) EFP 1258 'My goodness, Clara, how frightfully boring for you, how can you bear to listen to us'.

Cependant, ces deux énoncés relèvent de la modalité exclamative, et l'exclamation implique une «vive réaction», donc un «changement d'état »; or il en va de même de la «vive émotion » signifiée par l'adverbe frightfully. On ne peut ici prendre cet adverbe à la lettre, car la dématérialisation est manifeste, mais on peut s'interroger sur une éventuelle corrélation entre la valeur illocutoire exclamative et le choix de l'adverbe frightfully, d'autant que son remplacement par un adverbe de type maximizer semble difficile (?? completely, entirely, utterly interesting / boring).

3. Il y aurait ailleurs, sous une forme ou une autre, une « résistance du lexical » à la dématérialisation, résistance qui se manifeste à des degrés variables selon les contextes.

(9) A0D 741 'Mon dieu!'; said the little man, not apparently sure whether to be terribly angry or frightfully cross, '; Nicole!'

Le changement d'adverbe (terribly angry, frightfully cross) répond sans doute à un souci de variation lexicale, mais sachant que la colère peut provoquer la peur (relation de cause à effet), une interprétation littérale est envisageable avec lecture à rebours du syntagme adjectival.

La grammaticalisation des adverbes intensifs $\mathrm{A}, \mathrm{F}$ et $\mathrm{T}$ aurait cependant atteint un degré tel que leur signifié n'est généralement pas pris entièrement à la lettre. On peut à cet égard rappeler ce propos de Bolinger $(1972,54)$ cité par Guimier $(1986,62)$ : «An adverb modifying an adjective (...) tends to dilute its literal meaning in favor of more nearly straightforward intensification: horrible sensitiveness to criticism is more literal that horribly sensitive to criticism, terrible beauty is more literal than terribly beautiful. $»^{4}$

4. Les adverbes awfully, frightfully, terribly peuvent modaliser un adjectif qualificatif prototypique ${ }^{5}$ (hard), mais aussi un adjectif qui l'est moins (deserted), ainsi que certains « adjectifs limites » (alive) :

4 Roggero $(1979,115)$ écrit ceci : «Un nouveau pas amène à utiliser, pour la même signification de degré intensif, des adverbes toujours chargés quantitativement, mais qui ne sont plus du domaine de la dimension, comme fortement, pour lequel le trait de force disparaît aussi dans l'usage figuré. Il n'y a rien d'extraordinaire à dire d'un plat qu'il est fortement épicé, puisqu'on dit du poivre ou du piment qu'ils sont forts. Mais avec des associations comme fortement surpris ou fortement indigné le trait de force disparaît, et seul subsiste le trait central de la quantification. »

L'adjectif est ici considéré comme «prototypique» s'il est qualificatif (intersectif ou non ; plus ou moins 'neutre'), modifiable par des adverbes scalaires comme quite ou very, s'il admet les formes 
(10) A06 1912 The second year you realise that it's terribly, terribly hard.

(11) FS1 1734 The place looks terribly deserted.';

(12) EVC 2138 She was flesh and blood and terribly, terribly alive.

Paradis (1997) propose une classification tripartite des adjectifs en scalar, extreme et limit adjectives ${ }^{6}$. Un adjectif comme alive appartient clairement à la catégorie des limit adjectives, mais sous l'effet de la modalisation adverbiale, il est interprété ou recatégorisé en adjectif scalaire.

La grammaticalité de (12) montre donc la compatibilité entre un adverbe scalaire (terribly) et un adjectif limite (alive). En principe, un être humain est vivant ou ne l'est pas (alive/not alive), et ne peut être *rather/somewhat alive. Cependant, en opérant une portée sélective sur l'adjectif limite (sélectionnant un sème particulier), l'adverbe terribly induit une interprétation dérivée de l'adjectif, et l'adjectif limite devient adjectif scalaire. L'interprétation de l'adjectif alive ici est donc déterminée par l'appartenance catégorielle de terribly (booster, et non pas maximizer) ${ }^{7}$; de sorte que si, en termes d'incidence, l'adverbe intensif est subordonné à l'adjectif, ce rapport de subordination est inversé dans le processus interprétatif.

Les adverbes intensifs A, F, T se combinent non seulement avec les adjectifs prototypiques, avec certains adjectifs limites, mais aussi le cas échéant, avec un adjectif d'attitude (disposition adjectives) :

(13) EFP 179 I told her I was frightfully keen to carry on with French

(14) *I told her I was absolutely (entirely, totally, utterly) keen to carry on with French.

L'irrecevabilité de (14) montre que les 'disposition adjectives' (eager, keen, ready, willing, unwilling) sont difficilement compatibles avec les adverbes totalisants (totality modifiers).

5. Awfully, frightfully, terribly sont construits sur des bases adjectivales (awful, frightful, terrible) dont les racines nominales (awe, fright, terror)

comparative et superlative, et peut assumer indifféremment les fonctions épithète prénominale et attribut du sujet.

6 Les «adjectifs extrêmes » - terme utilisé par Paradis (1997, 56) - comme brilliant ou terrible, se présentent (eux aussi) en couples d'antonymes; ils entrent en relation paradigmatique avec des adjectifs dénotant des qualités intermédiaires entre deux extrêmes : entre brilliant et terrible, les qualités good et bad. L'adjectif extrême sera normalement modalisé par un maximizer comme absolutely ou utterly (absolutely brilliant/ disastrous), et non par un adverbe scalaire de type booster.

7 À l'inverse des maximizers et des approximators, les adverbes de type boosters (dont A, F, T) impliquent une absence de bornes limites dans l'échelle des qualités adjectivales (unboundedness). 
dénotent des «sentiments ${ }^{8}$. Sur l'échelle d'intensité des sentiments, ceux-ci se situent à l'extrémité d'un gradient. Cependant, $\mathrm{A}, \mathrm{F}$ et $\mathrm{T}$ sont des modificateurs scalaires (boosters), et non des modificateurs totalisants (maximizers) ; il s'en suit qu'ils construisent l'image du haut degré et non celle d'un dernier point indépassable.

Cruse $(1986,4)$ dit en substance la même chose en faisant valoir que l'intensifieur scalaire permet d'approcher l'extrémité du gradient uniquement de manière asymptotique ; et en effet, il n'y a pas de borne limite dans le degré de la qualité dénotée par l'adjectif scalaire. Ceci est confirmé $1^{\circ}$ ) par la récursivité de l'adjonction adverbiale avec terribly en (10) et (12): terribly, terribly hard; terribly, terribly alive; $2^{\circ}$ ) par la possibilité de modaliser l'adverbe scalaire frightfully avec l'adverbe intensif most :

(15) BMU 80 You know, in some ways I'm most frightfully sorry to leave

Most est compatible avec les adjectifs évaluatifs-subjectifs comme interesting, mais pas avec les adjectifs descriptifs comme short et long qui sont plus «neutres» tout en étant «relatifs» (non intersectifs): This is most interesting; *This bridge is most long. Cette observation est à mettre en parallèle avec la combinaison attestée most frightfully. Sachant que les adjectifs modalisés par most doivent comprendre une composante subjective, le contraste d'acceptabilité * most long / most interesting / most frightfully suggère le maintien dans l'adverbe intensif frightfully d'une composante sémantique en dépit de sa grammaticalisation.

6. Les adverbes intensifs scalaires de type booster (dont A, F et T) ne permettent donc pas de construire l'image d'un dernier point sur l'échelle scalaire ${ }^{9}$, contrairement aux maximizers ${ }^{10}$. Cette «incapacité » à construire l'image d'un dernier point peut être mise à profit, par exemple dans une structure de coordination $(\mathrm{C} 1$ and $\mathrm{C} 2)$ où la prédication adjectivale peut être déconstruite en $\mathrm{C} 2$ :

8 Comme le signale Guimier (1986, 61), les adverbes intensifs se divisent d'abord en deux catégories : $1^{\circ}$ ) il y a ceux construits sur une base adjectivale et qui évoquent le quantitatif (taille, mesure, poids, totalité, démesure etc.), par exemple, enormously, greatly, immensely, utterly; $2^{\circ}$ ) il y a ceux dont la racine exprime un sentiment quelconque (frightfully, horribly, terribly, terrifically, incredibly, wonderfully).

9 Paradis (2000, 148) écrit ceci : «Schematically degree modifiers map onto two different modes of construal, one of totality (an 'either-or' conception), e.g. completely and almost, and one of scalarity (a 'more-or-less' conception) e.g. very and fairly. This dichotomy is true of adjectives too. Some gradable adjectives are associated with a definite boundary, or totality, e.g. identical, true and dead, while others are unbounded and conceptualized according to a scale, long, good, fast and interesting. »

10 Dans les termes de Paradis $(2008,321)$ : «Maximizers (...) highlight the perfect match with a maximum or a BOUNDARY, eg. absolutely brilliant, completely full». 
(16) Our idealists were frightfully happy, but they were all the time reaching out for something to cotton on to. ( D.H.Lawrence, 'Things'.)

Cette nouvelle de D.H. Lawrence montre un couple d' «idéalistes » dont l'esprit matérialiste est progressivement dévoilé au fil des pages. Nous pouvons faire valoir ici que la «résistance du lexical» se manifeste par la participation de l'adverbe frightfully à la figure de l'oxymore dans la combinaison adverbeadjectif, dans un énoncé placé sous le sceau de l'ironie.

Si l'adverbe frightfully est pris au sens propre, à travers la figure de l'oxymore associant les notions de /fright/ et de /happiness/, nous comprenons que «Le bonheur de nos deux idéalistes était proprement effrayant». Cette interprétation est cependant exagérée étant donné la dématérialisation de l'adverbe intensif.

L'image du bonheur affichée dans la première coordonnée est déconstruite dans la deuxième coordonnée. Cette déconstruction de la représentation est semble-t-il permise par le statut de l'adverbe frightfully, adverbe scalaire indiquant le haut degré, et non un degré maximal qui serait un point de non retour. A l'appui de ce point de vue, la difficile recevabilité de (17) où l'adverbe scalaire a été remplacé par un maximizer :

(17) ?? Our idealists were absolutely/completely happy, but they were all the time reaching out for something to cotton on to.

L'énoncé (18) quant à lui montre que frightfully peut être remplacé par un adverbe exprimant l'incrédulité, incredibly/unbelievably, ou par l'adverbe extremely, qui lui aussi est un modificateur scalaire, tout en exprimant la notion d'extrémité :

(18) Our idealists were incredibly/unbelievably/extremely happy, but they were all the time reaching out for something to cotton on to.

Voici un exemple où l'adverbe d'incrédulité est une option naturelle :

(19) $\underline{\mathrm{CA} 0} 447$ Out of the window, Daisy admired the incredibly tidy garden

Un jardin peut manifester la qualité de /tidiness/ à un degré élevé ( $a$ very tidy garden), au point de susciter l'incrédulité (an incredibly tidy garden), mais rarement au point se susciter un sentiment de peur. Dans le groupe nominal complément du verbe admire, l'adverbe frightfully serait une option peu naturelle. Cependant, rien n'empêche dans un autre contexte de suggérer un rapport entre les notions de /tidiness/ et de /fright/ où le degré de 'tidiness' serait considéré comme trop élevé et de ce fait « effrayant » : a frightfully tidy garden. Le sens propre de l'adverbe serait alors réactivé. 
7. Le signifié lexical de awfully, frightfully, terribly est toujours disponible :

(20) JY6 3062 Has anyone ever told you you're frightfully serious?';

(21) ? Sérieux au point de faire peur

Avec une lecture à rebours du syntagme adjectival, un rapport de cause à effet peut ainsi être perçu (21). Il en va de même pour le syntagme nominal en (22) interprété en (23) :

(22) J0W 254 Ken playing Rodney, Hugh Paddick as Charles, two frightfully, frightfully Mayfair types, doing ridiculous things together like dressing up as red Indians when they took a canoeing holiday.

(23) ... des "Mayfair types" typiques au point de faire peur.

On voit en (22) que l'adverbe frightfully a été redoublé, comme terribly en (10) et (12), illustrant la récursivité syntaxique, et rappelant le caractère asymptotique de la modalisation scalaire.

Une préférence pour frightfully se retrouve également en (24), mais cette fois avec la possibilité d'une lecture métalinguistique du rôle de l'adverbe dans sa relation à l'adjectif, rappelant le lien entre la timidité et la peur :

(24) JA9 1188 Is it possible when we get a new member of, that, not that they're brought round, which I think must be frightfully intimidating, but that we are told, and therefore we can drop in and say, hello, I'm so and so, just to say hello.

Les adverbes awfully, frightfully et terribly sont des adverbes scalaires de type booster, signifiant le «haut degré » et figurant en discours plus ou moins dématérialisés. La « résistance du lexical » à la dématérialisation se vérifie dans la figure de l'oxymore (16); à travers la possibilité d'une lecture à rebours du couple adverbe-adjectif manifestant une relation de cause à effet $(20,22)$; et en (24) où le sens de l'adverbe se retrouve dans le signifié de l'adjectif. 
Christopher Desurmont

\section{Bibliographie}

Bolinger, Dwight, 1972. Degree Words, The Hague, Mouton.

CRuSE, Alan, 1986. Lexical Semantics, Cambridge. CUP.

GUIMIER, Claude, 1986. Syntaxe de l'adverbe anglais, Presses Universitaires de Lille.

PARADIS, Carita, 1997. Degree modifiers of adjectives in spoken British English. Lund Studies in English, Lund University Press.

- 2000. «It's well weird. Degree modifiers of adjectives revisited: the nineties», Corpora galore: analyses and techniques in describing English, ed by John M. Kirk. Amsterdam \& Atlanta: Rodopi. 147-160.

http://www.vxu.se/hum/publ/cpa/well_weird.pdf

- 2008. «Configurations, construals and change : expressions of DEGREE», English language and Linguistics, 12.2. 317-343.

http://www.vxu.se/hum/publ/cpa/degree.pdf

RogGERO, Jacques, 1979. «L'expression qualitative de la quantité », Sigma 4. 109-152. 